\title{
Colloidal Dynamics Near a Wall studied by Evanescent Wave Light Scattering: Experimental and theoretical Improvements and Methodological limitations
}

\author{
Peter Holmqvist, Jan K. G. Dhont and Peter R. Lang* \\ Forschugszentrum Jülich, Institut für Festkörperforschung, \\ Soft Matter Division, D-52425 Jülich, Germany
}

\begin{abstract}
The dynamics of colloidal spheres near to a wall is studied with an evanescent wave scattering setup that allows for an independent variation of the components of the scattering wave vector normal and parallel to the wall. The correlation functions obtained with this novel instrumentation are interpreted on the basis of an expression for their short time behavior that includes hydrodynamic interactions between the colloidal spheres and the wall. The combination of the evanescent wave scattering set-up and the exact expression for the short-time behavior of correlation functions allows for an unambiguous measurement of the particle mobility parallel and normal to the wall by means of light scattering. It is possible to measure the viscous wall drag effect on the dynamics of particles with radii as small as $27 \mathrm{~nm}$, where however, the method to reaches it's limits due to the low scattering intensities of such small particles.
\end{abstract}

PACS numbers: 82.70.Dd, 78.35.+c, 68.35.Fx

* email: p.lang@fz-juelich.de 


\section{Introduction}

The dynamics of colloids near a wall is expected to be hindered by hydrodynamic interactions. There has been a continuing debate whether this effect can be detected by evanescent wave dynamic light scattering (EWDLS), a technique which has been pioneered by the group of Ostrowsky ${ }^{1}$. In this paper we will show that the drag effect can be quantitatively investigated by EWDLS even for particles with radii significantly smaller than $100 \mathrm{~nm}$, if appropriate experimental and theoretical tools are applied. However we will also point out the limitations of the method in the range of very small particle sizes.

The problem of viscous wall drag has a history almost a century old ${ }^{2,3}$. The dynamics of particles close to a solid wall are different from the bulk dynamics due to hydrodynamic interactions between the colloidal particles and the wall. Expressions for the hydrodynamic interaction functions in the creeping flow limit with stick boundary conditions, which are widely accepted, have been published by Brenner and Goldman et al ${ }^{4,5}$ in the 1960's. They have shown that the hydrodynamic mobilities of a sphere normal and parallel to a planar wall tends to zero as the separation distance between the surface of the colloidal sphere and the wall becomes infinitely small. For a long period of time, there have been surprisingly few reports on experimental tests of this prediction. Most of these investigations deal with large spherical objects, sedimenting through a high viscous liquid towards the flat bottom of a vessel (e. g. $\left.{ }^{6-8}\right)$. In a more recent contribution Adamcyzk et al. describe experiments on the sedimentation of Nylon spheres with diameters ranging from 0.125 to 0.25 inch towards differently curved interfaces ${ }^{9}$. As a fluid they used silicon oil with a kinematic viscosity of 10 Stokes to achieve the creeping flow limit in the low Reynolds number regime. All experimental studies in this regime showed very good agreement with the theoretical predictions. The above experiments deal with spheres which are so large that Brownian motion is essentially absent.

Since low Reynolds numbers are a typical feature of colloidal particles in solution, Brenner's predictions are expected to also hold for these types of systems. For the investigation of the near-wall dynamics of Brownian particles, a technique is required, which probes the particles' mobility in a distance range smaller than several sphere diameters from the wall. Only with the advent of evanescent wave light scattering techniques and of powerful modern microcopy techniques this has become possible. Applying fluorescence microscopy combined with three dimensional particle tracking Khim et al. showed that Brenner's predictions for 
particle mobility parallel to a planar wall is valid for colloids ${ }^{10,11}$. In these studies, the mobility normal to the wall was found to be much smaller than expected. According to the authors this is due to particle wall interactions other than excluded volume and hydrodynamics. Using total internal reflection microscopy ${ }^{12,13}$ combined with an optical trap, Walz and Suresh studied the sedimentation of spherical polystyrene latex particles and found very good agreement with theory ${ }^{14}$. In a video microcopy experiment combined with optical tweezers Lin et al. showed that PMMA particles follow Brenner's prediction in both direction (parallel and normal to the wall), if the particle density is matched to the solvent and electrostatic interactions are sufficiently screened ${ }^{15}$. The particle sizes for which reliable dynamic data are obtainable by evaluating particle trajectories measured with microscopy techniques is limited to a minimum radius of 500 to $1000 \mathrm{~nm}$. Below this value correlation spectroscopy methods are preferable. Using fluorescence correlation spectroscopy Joly et al investigated the influence of surface wettability on the dynamics of $100 \mathrm{~nm}$ particles confined between two flat interfaces ${ }^{16}$. These authors found excellent agreement of the experimental data with hydrodynamic predictions for wettable surfaces. As expected, deviations occur for partially wettable surfaces, i. e. when the stick boundary conditions are violated.

EWDLS has been used several times to study the dynamics of colloidal spheres close to a glass-solution interface. However, the results which can be found in literature are contradicting. Feitosa et al. ${ }^{17}$ did not find experimental evidence for a difference between the near wall and the bulk particle dynamics. Garnier and Ostrowsky ${ }^{18}$ measured a reduction of the weighted average particle diffusion coefficient close to a wall, which was supported by Brownian dynamics simulations. However they could not experimentally detect the anisotropy of particle diffusivity in their EWDLS experiments. On the other hand Hosoda and coworkers ${ }^{19}$ showed clearly that particle mobility close to a wall is anisotropic for polystyrene colloids with a radius $R \gtrsim 400 \mathrm{~nm}$. For smaller particles $(R<200 \mathrm{~nm})$ the data by Hosoda et al. do not allow to distinguish unambiguously between bulk and near-wall dynamics. All these studies suffer from the fact that the diffusivity of the spheres parallel, $D_{\|}$, and normal, $D_{\perp}$, to the wall could not be measured separately. Furthermore, there was no theory for the time-auto correlation function of the scattered field, $g_{1}(t)$, taking into account hydrodynamic interactions, that is, the wall drag effect. We have cured this deficiency by constructing a novel EWDLS set-up and by deriving an exact expression for the short time relaxation rate $\Gamma$ of $g_{1}(t)$. Both issues have been 
discussed briefly in a very recent publication ${ }^{20}$. In this contribution, we will give the full derivation of the short time expansion of the correlation function up to the second cumulant $\Gamma_{2}$, including the effect of hydrodynamic and static particle wall interactions. With this expression we provide an analytical tool to interpret EWDLS-data from particles interacting with the surface, which was hitherto only possible using elaborate computer simulation techniques ${ }^{18}$. Furthermore we will describe the EWDLS set-up in detail. The latter allows to vary the magnitude of the scattering vector parallel, $Q_{\|}$, and normal, $Q_{\perp}$, to the interface independently and therefore to measure the diffusivities in both directions. We present and discuss EWDLS-data for two sizes of polystyrene spheres with $R=85 \mathrm{~nm}$ and $R=27 \mathrm{~nm}$ respectively in aqueous solution. The data from the former system agree quantitatively with Brenner's prediction, while for the latter the scattering intensities are very small. Therefore the error bars become too large to establish an accurate quantitative comparison with theoretical predictions.

\section{Brownian Motion Close to a Planar Interface and Light Scattering with} Evanescent Illumination

If a colloidal particle moves in the vicinity of a planar stationary wall, the surrounding fluid will cause a hydrodynamic interaction with the interface. The colloidal particle will feel a drag force which is larger than in the bulk. For low Reynolds numbers, i. e. in the creeping flow limit, Brenner calculated the multiplicative correction $\lambda_{\perp}{ }^{4}$ for the Stokes viscous force $\mathbf{F}_{\perp}=\mathbf{F}_{0} \lambda_{\perp}(z)$ exerted on a particle moving perpendicular to the wall, where $\mathbf{F}_{0}$ is the drag force in the bulk and $z$ is the shortest distance between the particle center and the wall,

$$
\begin{aligned}
\lambda_{\perp}(z) & =\frac{4 \sinh \alpha}{3} \sum_{n=1}^{\infty} \frac{n(n+1)}{(2 n-1)(2 n+3)} \\
& \times\left[\frac{2 \sinh [(2 n+1) \alpha]+(2 n+1) \sinh [2 \alpha]}{(2 \sinh [(n+1 / 2) \alpha])^{2}-((2 n+1) \sinh \alpha)^{2}}-1\right] .
\end{aligned}
$$

where $\alpha=\cosh ^{-1}(z / R)$. For large $z$ it is sufficient to include only the first summand in eq. 1 and to approximate $\sinh \alpha$ by $\exp \{\alpha\} / 2$ which yields,

$$
\lambda_{\perp}(z)=\left(1-\frac{9}{4} \exp \{-\alpha\}\right)^{-1} .
$$

With the definition $\cosh \alpha=z / R$ we may further use $\exp \{-\alpha\} \approx R / 2 z$ to get an approxi- 
mate expression for the correction factor

$$
\lambda_{\perp}(z)=\left(1-\frac{9}{8} \frac{R}{z}\right)^{-1} .
$$

This approximation, which coincides with the older approximative expression by Lorentz ${ }^{2}$, deviates by $20 \%$ from the exact expression at $z / R=1.5,10 \%$ at $z / R=2$ and less than $1 \%$ at $z / R=3$.

For the case of a sphere moving parallel to a wall, the Stokes force may similarly be expressed as the product of the bulk force and a correction term, $\mathbf{F}_{\|}=\mathbf{F}_{0} \lambda_{\|}(z)$. However, there is no exact analytical expression for $\lambda_{\|}$. The commonly applied approximation,

$$
\lambda_{\|}(z)=\left[1-\frac{9}{16} \frac{R}{z}+\frac{1}{8}\left(\frac{R}{z}\right)^{3}-\frac{45}{256}\left(\frac{R}{z}\right)^{4}-\frac{1}{16}\left(\frac{R}{z}\right)^{5}\right]^{-1},
$$

is due to Faxén ${ }^{3}$. Comparing this expression with numerical calculations by O'Neill ${ }^{21}$ Goldman et al. ${ }^{5}$ shows that it deviates less than $10 \%$ for $z / R>1.04$ from the numerical results and that it describes them essentially exact for $z / R>1.4$. The Brownian motion of colloidal particles with a bulk diffusion coefficient $D_{0}$ will be hindered in the ultimate vicinity of a wall due to the increased drag forces. As a consequence, the diffusivities parallel $D_{\|}(z)=D_{0} / \lambda_{\|}(z)$ and normal $D_{\perp}(z)=D_{0} / \lambda_{\perp}(z)$ to the wall depend on the particle's $z$-position with different functional forms.

The near-wall dynamics of Brownian particles can be studied by EWDLS. If a Laser beam is totaly reflected from the interface between a glass with refractive index $n_{1}$ and a solution with refractive index $n_{2}<n_{1}$, as sketched in Fig. 1, an evanescent wave is formed on the solution side of the interface. The electric field strength of this wave decays exponentially with the distance from the interface as,

$$
\mathbf{E}=\mathbf{E}_{0} \exp \left\{-\frac{\kappa}{2} z\right\}
$$

where $\kappa / 2=2 \pi \sqrt{\left(n_{1} \sin \alpha_{i}\right)^{2}-n_{2}^{2}} / \lambda_{0}$ is the inverse penetration depth of the evanescent field strength, with $\lambda_{0}$ the vacuum wavelength of the laser and $\alpha_{i}$ the angle of incidence. Particles which are close enough to the interface will scatter light from the evanescent wave. The scattered intensity can be correlated to study particle dynamics. With evanescent illumination which is homogeneous in lateral directions at constant $z$, the scattered field at a given time $t$ from an ensemble of $N$ identical particles is,

$$
\mathbf{E}_{S} \propto \sum_{j}^{N} \exp \left\{-\frac{\kappa}{2} z_{j}\right\} \exp \left\{\imath \mathbf{Q} \cdot \mathbf{r}_{j}\right\}
$$


Here the scattering vector is the difference between the wave vector of the scattered and the evanescent wave, $\mathbf{Q}=\mathbf{k}_{S}-\mathbf{k}_{e}$ and $\mathbf{r}_{j}$ defines the position of the $j^{\text {th }}$ particle. Due to translational invariance in lateral directions parallel to the wall, the Siegert relation for the scattered intensity-auto correlation

$$
g_{2}(t)=1+\left|g_{1}(t)\right|^{2}
$$

holds whenever the scattered wave vector parallel to the wall is non-zero. The quantity of interest to extract dynamic information from a scattering experiment is therefore the time-auto correlation function of the scattered field $g_{1}(t)=\langle E(0) E(t)\rangle$, with $E$ the scattered electric field strength. This field-correlation function is related to the probability density function (PDF) $P\left(\mathbf{r}_{0}\right)$ for the position coordinate of the colloidal sphere and to the conditional $\operatorname{PDF} P\left(\mathbf{r}_{0}, \mathbf{r} \mid t\right)$ of finding a particle at position $\mathbf{r}$ at time $t$ which was at the position $\mathbf{r}_{0}$ at $t=0$ by

$$
g_{1}(t)=\int_{z>R} d \mathbf{r}_{0} \int_{z>R} d \mathbf{r} P\left(\mathbf{r}_{0}, \mathbf{r} \mid t\right) P\left(\mathbf{r}_{0}\right) \exp \left\{-\frac{\kappa}{2}\left(z_{0}+z\right)\right\} \exp \left\{\imath \mathbf{Q} \cdot\left(\mathbf{r}_{0}-\mathbf{r}\right)\right\} .
$$

Apart from a normalization factor $P\left(\mathbf{r}_{0}\right)$ is equal to the Boltzumann-factor of the interaction potential between the particle and the wall, i. e. $P\left(\mathbf{r}_{0}\right) \propto \exp \{-\beta V(z)\}$. The spatial integrations range over $z$-values larger than the radius $R$ of the colloidal particle, since the PDF is zero for smaller values for $z$. In a bulk solution of non-interacting particles the PDF is a solution of Fick's second law. In the vicinity of a wall the complex dependence of the particle mobility on the separation distance has to be taken into account, with which the equation of motion becomes,

$$
\frac{\partial P\left(\mathbf{r}_{0}, \mathbf{r} \mid t\right)}{\partial t}=\frac{\partial}{\partial z}\left(D_{\perp}(z) \frac{P\left(\mathbf{r}_{0}, \mathbf{r} \mid t\right)}{\partial z}\right)+D_{\|}(z)\left(\frac{\partial^{2}}{\partial x^{2}}+\frac{\partial^{2}}{\partial y^{2}}\right) P\left(\mathbf{r}_{0}, \mathbf{r} \mid t\right)
$$

So far this equation of motion has not been solved analytically. Here, we calculate an expression for the short time behavior of the correlation function,

$$
g_{1}(t)=\exp \left\{-\Gamma t+\mathcal{O}\left(t^{2}\right)\right\}
$$

the details of which are given in the Appendix. For particles which interact with the wall only by hard core repulsion, the initial relaxation rate $\Gamma$ is given by,

$$
\Gamma=\kappa \int_{R}^{\infty} d z \exp \{-\kappa(z-R)\}\left[D_{\|}(z) Q_{\|}^{2}+D_{\perp}(z)\left(Q_{\perp}^{2}+\frac{\kappa^{2}}{4}\right)\right] .
$$


Here interactions between colloidal particles are neglected, that is, this expression is valid for very dilute suspensions. Here, $Q_{\|, \perp}$ are the magnitude of the scattering vector component parallel and normal to the interface. An analogous expression can be derived for the second cumulant, which now contains the derivative $\partial D_{\perp}(z) / \partial z$ of the mobility normal to the interface (see eq. 37 in the appendix).

The expression for the first cumulant is conveniently rewritten in the following form,

$$
\Gamma=Q_{\|}^{2}\left\langle D_{\|}\right\rangle(\kappa)+\left(Q_{\perp}^{2}+\frac{\kappa^{2}}{4}\right)\left\langle D_{\perp}\right\rangle(\kappa),
$$

where "the mean diffusivities" are defined as,

$$
\left\langle D_{\|, \perp}\right\rangle(\kappa)=D_{0} \kappa \int_{R}^{\infty} d z \exp \{-\kappa(z-R)\} / \lambda_{\|, \perp}(z),
$$

where $D_{0}$ is the Einstein bulk diffusion coefficient. EWDLS thus measures the Laplace transform of the hydrodynamic interaction functions, where the inverse penetration depth is the Laplace-variable.

The penetration-depth dependence of the mean diffusivities can be obtained on the basis of the Brenner-Lorentz and Faxén's approximation. Introducing eqs. 3 and 4 for $\lambda_{\|, \perp}$ into eq. 13 we obtain,

$$
\begin{aligned}
\left\langle D_{\|}\right\rangle(\kappa) \cong & D_{0}\left(\left[-\frac{9}{16} \mathcal{X}+\frac{1}{16} \mathcal{X}^{3}+\frac{15}{512} \mathcal{X}^{4}-\frac{1}{384} \mathcal{X}^{5}\right]\right. \\
& \times \exp \{\mathcal{X}\} E_{1}(\mathcal{X}) \\
+ & \left.1-\frac{3}{256} \mathcal{X}-\frac{43}{1536} \mathcal{X}^{2}-\frac{49}{1536} \mathcal{X}^{3}+\frac{1}{384} \mathcal{X}^{4}\right)
\end{aligned}
$$

and,

$$
\left\langle D_{\perp}\right\rangle(\kappa) \cong D_{0}\left(1-\frac{9}{8} \mathcal{X} \exp \{\mathcal{X}\} E_{1}(\mathcal{X})\right)
$$

with $\mathcal{X}=\kappa R$, the exponential integral $E_{1}(\mathcal{X})=\int_{1}^{\infty} d z^{\prime} \exp \left\{-z^{\prime} \mathcal{X}\right\} / z^{\prime}$ and $z^{\prime}=z / R$. We made use of the general property of exponential integrals,

$$
\begin{aligned}
E_{n}(a) & =\int_{1}^{\infty} d x \frac{1}{x^{n}} \exp \{-a x\} \\
& =\frac{(-a)^{n-1}}{(n-1) !} E_{1}(a)+\exp \{-a\} \sum_{j=1}^{n-1}(-1)^{j+1} \frac{(n-1-j) !}{(n-1) !} a^{j-1}
\end{aligned}
$$

to obtain eq. 14. $E_{1}(a)$ can be expanded as,

$$
E_{1}(a) \cong-\gamma-\ln (a)-\sum_{n=1}^{\infty} \frac{(-a)^{n}}{n ! n}
$$


where $\gamma=0.57721566$ is the Euler-Mascheroni constant $^{22}$.

The exact expression for the first cumulant (eqs. 12 and 13) has not been derived before. It allows for an unambiguous interpretation of measured correlation functions in terms of hydrodynamic interaction functions, provided that $Q_{\perp}$ and $Q_{\|}$can be varied independently in an experiment.

\section{Experimental}

EWDLS-setup: According to eq. 12, the initial relaxation rate of the field-auto correlation function depends linearly on $Q_{\|}^{2}$ and $\left(Q_{\perp}^{2}+\kappa^{2} / 4\right)$. For the model-free determination of the mean diffusivities $\left\langle D_{\|, \perp}\right\rangle$ in an EWDLS-experiment it is therefore required to change the two scattering vector components independently of each other. As sketched in Fig. 1 the magnitudes of the scattering vector components are given by $Q_{\|}=2 \pi \sqrt{1+\cos \alpha_{r}^{2}-2 \cos \alpha_{r} \cos \theta} / \lambda$ and $Q_{\perp}=2 \pi \sin \alpha_{r} / \lambda$ respectively and the mag-

nitude of the total scattering vector is $Q=\sqrt{Q_{\|}^{2}+Q_{\perp}^{2}}$. In all EWLS-instruments described so far ${ }^{1,17,19,23,24}$, it is only possible to change $\alpha_{r}$, which will vary both $Q_{\|}$and $Q_{\perp}$ simultaneously. We therefore used a three axis goniometer as the mechanical basis for our set up, which was custom-made by Huber Diffraktionstechnik, Rimsting, Germany. The instrument is equipped with a HeNe-Laser with a power output of $35 \mathrm{~mW}$ and $\lambda_{0}=632.8 \mathrm{~nm}$ as a light source, which is mounted on the source goniometer arm. The angle of incidence can be varied in the range $60 \leq \alpha_{i} \leq 180$ degrees. Where angles of $\alpha_{i} \geq 90$ degrees are needed for alignment purposes only. During experiments, the primary beam is polarized parallel to the plane of incidence by means of a $\lambda / 2$-plate and a polarizer (Bernhard Halle Nachfl, Berlin Germany). Varying the angle of incidence, the penetration depth of the evanescent wave can be changed approximately in the range $100 \mathrm{~nm}<2 / \kappa<1 \mu \mathrm{m}$. The upper limit is mainly set by focusing of the incoming beam in the hemispherical bottom part of the sample cell and the fact that the penetration depth diverges at the critical angle of total reflection.

The scattered light is collected with a monomode optical fiber (OZ-Optics, Ottawa, Canada) and detected with an ALV-APD avalanche diode by ALV-Laservertriebsgesellschaft, Langen, Germany. The detecting unit can be moved on a section of a spherical surface by variation of the angles $\alpha_{r}$ and $\theta$ with two goniometers which are mounted normal to each other. In our experiments we explore scattering vectors which point into the upper right 
quarter (looking beam down) of the sphere. In general we limit the scattering angles to the region were $\alpha_{r}+\theta>30$, because at smaller angles statically scattered light from surface roughnesses or impurities becomes increasingly interfering. The upper limit of scattering angle is set by the average scattered intensity. Usually $\alpha_{r} \leq 60$ and $\theta \leq 120$ because the scattered intensities are to small to record correlation functions at larger angles. The TTL output of the avalanche diode is processed with a multiple tau correlator, ALV-6010. The shortest delay time which is accessible with this detector correlator combination is $1 \mu$ s due to correlated noise of the avalanche diode at shorter times.

The sample cell (custom-made by Hellma GmbH, Müllheim, Germany) consists of a hemispherical lens as the bottom part, made of SF10 glass, which has an index of refraction $n_{1}=1.723$ at $\lambda_{0}=632.8 \mathrm{~nm}$. The aqueous sample solution is contained in a hemispherical dome sitting on top of the lens. The primary beam is reflected off the interface between the glass and the solution. The lens and the dome have different radii but a common center. The instrument has to be aligned such that the primary beam always hits the center of the sample cell, which in turn has to be located in the intersection point of the goniometer axes. Further the reflecting interface has to be normal to the plane which is spanned by the primary and the reflected beam. To provide the degrees of freedom required for this alignment, the sample cell is mounted on top of a combination of a turntable, a vertical $z$-stage, a horizontal $x y$-stage and two circle segment cradles.

Samples and preparation: Aqueous solutions of two types of polystyrene latex spheres (Interfacial Dynamics Corp., Portland, USA) with radii of $R=85 \mathrm{~nm}$ and $R=27 \mathrm{~nm}$ respectively have been investigated. Both samples are charge stabilized by sulfonate surface groups and were diluted from their stock solutions with $0.01 \mathrm{M}$ salt solution to a volume fraction of $2 \cdot 10^{-4}$. At these conditions the particles may be regarded as hard spheres, since the Debye screening length is in the range of $3 \mathrm{~nm}$ while the mean inter-particle distance is of the order of several thousand nm. The volume fraction was chosen such that the scattered intensity was sufficiently high in the EWDLS-experiments and no variation of the particles' bulk diffusion coefficient could be detected by conventional dynamic light scattering (DLS), if the volume fraction was further decreased. For the reference DLS-measurements we used a commercial light scattering setup by ALV. 
Data analysis: For the data analysis of conventional bulk DLS data usually a simple quadratic relation between the field and the intensity correlation function is assumed. In the case of EWDLS-experiments this does not necessary hold, because there the scattered light consists unavoidably of two contributions, light scattered from the sample particles and light scattered from surface roughnesses and small impurities. The latter may be a purely static contribution, which leads to a mixing of homodyne and heterodyne detection. In this case the more general relation ${ }^{25}$

$$
g_{2}(t)=1+2 C_{1} g_{1}(t)+\left(C_{2} g_{1}(t)\right)^{2}
$$

for the correlation function of the scattered intensity has to be applied. Here, $C_{2}=1-$ $\sqrt{1-A}$ and $C_{1}=C_{2}-C_{2}^{2}$, with $A$ the intercept of intensity-auto correlation function $g_{2}(t)$. In many cases EWDLS intensity-auto correlation functions exhibit a very slow decay at large times. The physical origin of this slow relaxation is not clear yet. Garnier et al conjecture that it is due to a slow reversible adsorption of the particles to the wall due to van der Waals attraction ${ }^{18}$. Since these slow modes are well separated from the relaxation rates of interest, as shown in Fig. 2, we approximate these slow modes by an additional baseline $B_{1}$ to $g_{1}(t)$. Further, a detailed analysis of the short time expansion of $g_{1}(t)$ shows that the second cumulant may become non-negligible at very short delay times. If we define a maximum time $t_{\max }$ up to which a first order approximation is valid by $\mid \Gamma t>10 \Gamma_{2} t^{2} / 2$, i. e. $t_{\max }=0.2 \Gamma / \Gamma_{2}$, we find a complex dependence of $t_{\max }$ on the particle size, the penetration depth and the scattering vector. As shown in Fig. $3 t_{\max }$ may become smaller than $0.1 \mathrm{~ms}$ for particles with $R=27 \mathrm{~nm}$. At this delay time the correlation function will have relaxed only to about $0.9 A$ and $t_{\max }<<1 / \Gamma$. We therefore chose to non-linear least squares fit the experimental correlation functions to eq. 18, where $g_{1}(t)$ is approximated by a cumulant expansion to second order in time

$$
g_{1}(t)=\left(1-B_{1}\right) \exp \left\{-\Gamma t+\frac{\Gamma_{2} t^{2}}{2}\right\}+B_{1} .
$$

According to eqs. 18 and $19, B_{1}$ is related to $B_{2}$, the baseline of $g_{2}(t)$, by $B_{1}=$ $\sqrt{\left(C_{1} / C_{2}^{2}\right)^{2}+B_{2} / C_{2}^{2}}-C_{1} / C_{2}^{2}$. Consequently there are four fit parameters $A, \Gamma, \Gamma_{2}$ and $B_{2}$. An erroneous baseline value will lead to a deviation of $\Gamma$ from its true value, due to a 
normalization error. We therefore fitted the experimental curves repeatedly starting from a manually chosen number of data points, $N_{p}$, at which the model function was evaluated. When a single fit had converged, $N_{p}$ was reduced by two and the remaining data were fitted again. With this procedure it is possible to identify a limited range of $N_{p}$ 's where the values of $B_{2}$ and $\Gamma$ are essentially independent of $N_{p}$. These $\Gamma$-values from this range are compared to theory in the Results and Discussion section. Error bars for the individual parameters were defined as the variation necessary to increase the sum of the squared deviations by $30 \%$ while all other parameters were fixed. In order to minimize the influence of higher order terms we applied a further constraint to the fitting algorithm, i. e. only data points for which $|\Gamma t|>\Gamma_{2} t^{2} / 2$ and $\Gamma_{2} t^{2} / 2<1$ were evaluated.

\section{Results and Discussion}

With evanescent wave light scattering it is not possible to measure the diffusivity of the particles at a well defined distance from the wall. According to eq. 13 the mean diffusivities are averaged along the $z$-direction over the entire depth which is illuminated by the evanescent wave. In Fig 4 we plot these averaged diffusivities normalized to the bulk diffusion coefficient, $\left\langle D_{\|, \perp}\right\rangle / D_{0}$ versus the normalized penetration depth $\xi=2 /(\kappa R)$ to compare the approximate expressions given by eqs. 14 and 15 with the exact solutions of eq. 13 obtained by numerical integration. For the case of $\left\langle D_{\perp}\right\rangle$, the value of the integrand depends on the number of terms, $n_{\max }$, which is taken into account for the calculation of $\lambda_{\perp}$ according to eq. 1. From the inset of Fig. 4 it is evident that for $n_{\max }>10$ the solutions become indistinguishable. Therefore the corresponding curve in the main plot was calculated with $n_{\max }=50$.

In the case of $\left\langle D_{\|}\right\rangle$the approximation by eq. 14 is very accurate for $\xi \geq 2$, while $\left\langle D_{\perp}\right\rangle$ is underestimated by $10 \%$ at $\xi \approx 2$ as compared to a numerical integration of eq. 13 . This deviation decreases to about $2 \%$ at $\xi=10$ and virtually vanishes at $\xi=30$. Both approximations fail completely at penetration depths below two particle radii. Therefore eqs. 14 and 15 are good approximations for the range of $\xi$ covered in the experiments which will be discussed in the following.

According to eq. 12, the scattering vector dependence of the first cumulant can be in-

terpreted in terms of averaged diffusivities. An accordingly intuitive interpretation of the functional form of the second cumulant is not possible. We thus limit the further discussion to the analysis of the first cumulant. To determine the mean diffusivities experimentally, the 
measured values of $\Gamma$ were plotted versus $Q_{\|}^{2}$ or $\left(Q_{\perp}^{2}+(\kappa / 2)^{2}\right)$ respectively as is shown Fig. 5 for the data from the sample with $R=85 \mathrm{~nm}$ recorded at a penetration depth of $2 / \kappa=234$ nm. From the linear least squares fits we obtained the averaged mobilities according to eq. 12 , i. e. $\left\langle D_{\|}\right\rangle$is the slope and $\left\langle D_{\perp}\right\rangle$ is the intersect of the linear dependence of $\Gamma$ on $Q_{\|}^{2}$ and vice versa, if $\Gamma$ is plotted versus $\left(Q_{\perp}^{2}+(\kappa / 2)^{2}\right)$. As expected from the theoretical predictions, the slope of the $\Gamma$ vs. $Q_{\|}^{2}$ data, i. e. the mean diffusivity parallel to the wall, is significantly larger than $\left\langle D_{\perp}\right\rangle$, that is $\partial \Gamma / \partial\left(Q_{\perp}^{2}+\left(\kappa^{2} / 4\right)\right)$.

According to eqs. 14 and 15 the mean diffusivities are expected to approach the bulk diffusion coefficient at sufficiently small values of $\mathcal{X}=\kappa R$ which corresponds to large values $\xi$. To investigate the dependence of the mean diffusivities on the penetration depth we performed experiments at four different angles of incidence. The resulting values of $\left\langle D_{\|, \perp}\right\rangle / D_{0}$ are plotted versus $\xi$ in Fig. 6 together with the theoretical predictions for various interaction potentials between the particles and the wall. In addition to the hard-wall interaction potential, we used an electrostatic interaction potential which in the Debye-Hückel limit may be approximated by,

$$
V_{e r}(z)=B_{e s} \exp \left\{-\kappa_{D}(z-R)\right\}
$$

where $\kappa_{D}^{-1}$ is the Debye screening length which in all experiments was adjusted to $3 \mathrm{~nm}$ by addition of electrolyte. The amplitude $B_{e s}$ scales with the particle radius and depends on the charge density of the particle surface and the wall surface. It is in general difficult to determine but it may amount to $B_{e s}=10^{4} k_{B} T$ for large particles ${ }^{12}$. In the present case a value of $B_{e s} \approx 100-200 k_{B} T$ is more realistic. Broken lines in Fig. 6 correspond to finite values of $B_{e s}$ while the full line represents the case without electrostatic interaction, i. e. $B_{e s}=0$. The latter was calculated by numerical integration of eq. 13 while the curves for finite $B_{e s}$ were calculated from eq. 33 of the Appendix. For the sake of completeness we have also added a curve which corresponds to a weakly attractive electrostatic interaction, i.e. $B_{e s}=-7 k_{B} T$. Note that in this representation the curves which include electrostatic interaction in general will not coincide for different particle radii, because the range of the potential scales differently with the particle radius. However for $\xi \gtrsim 15$ the curves for $R=85$ $\mathrm{nm}$ and $R=27 \mathrm{~nm}$ can hardly be distinguished. For the sake of clarity we therefore chose to present only the theoretical curves for $R=85 \mathrm{~nm}$.

Furthermore, in Fig. 7 we compare our experimental data to theoretical curves calculated for repulsive electrostatic potentials, where we kept $B_{e s}$ constant and changed $\kappa_{D}^{-1}$. It 
is worth to note that repulsive interactions of the spheres with the wall at constant screening length have a much weaker effect on the mean diffusivities than attractive interactions. In the latter case $\left\langle D_{\|, \perp}\right\rangle$ decrease drastically with increasing interaction strength and the anisotropy increases in the same direction. This may provide an explanation for the slow particle dynamics normal to a wall which was observed by Khim etal ${ }^{10,11}$. In a particle tracking experiment combined with fluorescence microscopy, these authors found that particle mobility parallel to the wall was in agreement with Brenner's predictions, while the normal component was unexpectedly small. They conjecture this to be due to static interactions between the particles and the wall.

On the other hand, changes of the electrostatic screening length, i. e. the range of the potential, at constant charge parameter strongly affects the dynamic properties of the particles. With increasing range of the potential, the near wall diffusivities approach the bulk diffusion coefficient. For $\kappa_{D}^{-1} / R=10$, (which corresponds roughly to pure water as the solvent for particles with $R>90 \mathrm{~nm}$ ) the near wall dynamics is virtually indiscernible from the bulk behavior. This is intuitively expected, since a long range repulsive potential will effectively repel the particles from the interfacial region, where the hydrodynamic drag effect is active on the colloids. Quantitatively this has been observed by Garnier and Ostrowsky ${ }^{18}$, who have shown by EWDLS-experiments and Brownian dynamics simulations, that the weighted average particle diffusion coefficient close to a wall increases with decreasing ionic strength of the solution, i. e. with increasing range of the electrostatic repulsion. However, to interpret their data these authors had to apply Brownian dynamics simulations. In this sense, our analytical expression for $\Gamma$ greatly facilitates the analysis of EWDLS-data from particles interacting with the wall, because it requires only simple numerical integration.

Our experimental data obtained from the particles of $R=85 \mathrm{~nm}$ coincide equally well with the predicted curves for $B_{e s}=100 k_{B} T$ and without electrostatic interaction. Thus, the particles may be treated as hard spheres under the present experimental conditions. The experimental results for the two highest penetration depths agree slightly better to the hard particle prediction than reported previously ${ }^{20}$. This is due to the improved data analysis scheme including the second cumulant. For the two lowest penetration depths, this did not change the result observably. The resulting mean diffusivities are significantly smaller than $D_{0}$, even for the highest penetration depth, $2 / \kappa=551 \mathrm{~nm}$. To obtain data at larger $\xi$ we performed additional experiments on a sample with $R=27 \mathrm{~nm}$ at three different penetration 
depths, $2 / \kappa=225,447$ and $835 \mathrm{~nm}$. The resulting data for the mean diffusivities are plotted as open symbols in Fig. 6. EWDLS-experiments with such small particles are extremely difficult because of the reduced scattered intensity. Therefore, the experimental uncertainties are too large to allow for an accurate quantitative verification of theoretical predictions. It appears, that despite the sophisticated instrumentation and the elaborate data analysis we have arrived at the performance limits of EWDLS with the particles of $R=27 \mathrm{~nm}$.

Note that predicted values for the mean diffusivities are in the range of $\left\langle D_{\|}\right\rangle \approx 0.9 D_{0}$ and $\left\langle D_{\perp}\right\rangle \approx 0.8 D_{0}$ even at $\xi=30$. This is due to the different weighting of the particles by the exponentially decaying field strength of the evanescent illumination. The closer a particle is to the wall the less mobile it is, and the higher the strength of the evanescent field it experiences. Therefore, less mobile particles contribute with a higher weight to the scattered intensity than particles with a higher mobility, which are further away from the wall. As can be seen from Fig. $6,\left\langle D_{\|, \perp}\right\rangle / D_{0}$ increase only very gradually at large $\xi$. In fact, eqs. 14 and 15 show that $\left\langle D_{\|}\right\rangle>0.95 D_{0}$ only for $2 / \kappa R>70$, which is experimentally not accessible. It is therefore important to distinguish between the effect of the wall viscous drag on the particle mobility and the averaging effect which is inherent in the experimental technique. This is most effectively done by considering the limiting case where no drag effect is present. In this case $\lambda_{\|, \perp}=1$ and the expression for the initial relaxation rate of $g_{1}(t)$ reduces to,

$$
\Gamma^{0}=D_{0}\left[Q_{\|}^{2}+\left(Q_{\perp}^{2}+\frac{\kappa^{2}}{4}\right)\right] .
$$

Consequently, in this case the determination of $\partial \Gamma / \partial\left(Q_{\|}^{2}\right)$ and $\partial \Gamma / \partial\left(Q_{\perp}^{2}+\left(\kappa^{2} / 4\right)\right)$ along our scheme of data analysis, would yield $D_{0}$ independently of the penetration depth. In turn this means that any significant deviation of $\left\langle D_{\|, \perp}\right\rangle / D_{0}$ from unity has to be ascribed to the viscous drag effect the wall exerts on the particles, if no further particle-wall interactions are active as in the present case $^{20}$. Repulsive interactions of the particle with the wall would mask the drag effect in an EWDLS experiment, according to our calculations. On the other side, the anisotropy of the mean diffusivities is expected to become much stronger then predicted by eqs. 14 and 15, if additional attractive forces between the wall and the colloids are present. An experimental example for this was reported by Kihm et al. ${ }^{11}$ who conjectured that an observed additional decrease of the particle mobility normal to the interface was caused by electrostatic or electro-osmotic effects. 
Conclusions We present an improved and extended approach to study Brownian motion of spherical particles close to a planar interface. The major experimental improvement consists of the possibility to change the scattering vector components parallel and normal to the interface independently of each other. This allows for the determination of the particle mobility components in both directions. Furthermore, we have improved the theoretical basis of EWDLS by deriving an expression for the short-time behavior of the auto correlation function of the scattered field, which includes hydrodynamic and static interaction of the colloid with the wall. In this paper we restricted the discussion to the special case of purely hydrodynamic interaction. The combination of an exact expression for the initial decay rate for data analysis and the novel experimental possibilities, allows for the investigation of the near-wall dynamics of particles with a radius as small as $27 \mathrm{~nm}$. It is worth to note that the Brownian motion of such small particles follows the predictions for the hydrodynamic interaction with a wall, which was originally derived for the stationary motion of a sphere towards a wall through a highly viscous liquid. With particle radii in the range of $25 \mathrm{~nm}$ the method appears to encounter it's limitations. Nevertheless, the present state-of-the-art of evanescent wave scattering opens the way to study more complicated systems. Especially as our theoretical expression for the short time behavior of the correlation function allows to easily interpret data from systems with static interaction between particle and wall, which hitherto was possible only with involved computer simulation techniques. This will greatly facilitate the investigation of systems where the static interactions between colloidal spheres and the wall are more complex such as depletion interaction or steric repulsion due to polymer coatings.

\section{Acknowledgement}

We thank M. McPhie for helpful discussions, especially concerning the approximate expressions for the averaged particle mobilities.

\section{Appendix: Short time expansion of $g_{1}(t)$}

Consider spherical colloids with a concentration that is small enough to neglect inter-colloidal particle interactions. The equation of motion for the conditional PDF of the position coor- 
dinate of a colloidal sphere is,

$$
\frac{\partial P\left(\mathbf{r}_{0}, \mathbf{r} \mid t\right)}{\partial t}=\widehat{\mathcal{L}} P\left(\mathbf{r}_{0}, \mathbf{r} \mid t\right) \quad(z \geq R, t \geq 0)
$$

where $\widehat{\mathcal{L}}$ is the Smoluchowski operator,

$$
\widehat{\mathcal{L}}(\cdots)=\frac{\partial}{\partial z}\left(D_{\perp}(z)\left\{\frac{\partial}{\partial z}(\cdots)+\beta(\cdots) \frac{d}{d z} V(z)\right\}\right)+D_{\|}(z)\left(\frac{\partial^{2}}{\partial x^{2}}+\partial y^{2}\right)(\cdots)
$$

where $(\cdots)$ stands for an arbitrary phase function. Here, $V(z)$ is the potential between a colloidal sphere and the wall, in addition to the hard-core interaction. This potential may be due to, for example, charges on the wall and the colloidal sphere. In comparison to eq. 9, the above form of the Smoluchowski operator includes a term that accounts for interactions with the wall other than the hard-core interaction. The distance $z$ from the wall in eq. 22 is always larger than the radius $R$ of a colloidal sphere, since the wall is assumed to be inpenetrable for the colloidal sphere. The formal solution of eq. 22 under the initial condition that the colloidal sphere is at $\mathbf{r}_{0}$ at time $t=0$ reads,

$$
P\left(\mathbf{r}_{0}, \mathbf{r} \mid t\right)=\exp \{\widehat{\mathcal{L}} t\} P(\mathbf{r}, t=0)=\exp \{\widehat{\mathcal{L}} t\} \delta\left(\mathbf{r}-\mathbf{r}_{0}\right)
$$

where $\delta$ is the delta-distribution and $P(\mathbf{r}, t=0)$ is the unconditional PDF of finding a particle at position $\mathbf{r}$ at $t=0$. The operator exponential is defined through its Taylor expansion. The correlation function in eq. 8 can thus be written as,

$$
g_{1}(t) \propto \int_{z_{0}>R} d \mathbf{r}_{0} \int_{z>R} d \mathbf{r} P\left(\mathbf{r}_{0}\right) \exp \left\{\imath \mathbf{Q} \cdot\left(\mathbf{r}_{0}-\mathbf{r}\right)\right\} \exp \left\{-\frac{\kappa}{2}\left(z_{0}+z\right)\right\} \exp \{\widehat{\mathcal{L}} t\} \delta\left(\mathbf{r}-\mathbf{r}_{0}\right) .
$$

Apart from a normalization constant, the latter PDF is equal to,

$$
P\left(\mathbf{r}_{0}\right) \propto \exp \{-\beta V(z)\} \quad(z \geq 0)
$$

Note that the integrations in eq. 25 range over $z$-values larger than the radius $R$ of the colloidal sphere, since the colloidal particles can not penetrate the wall, that is, since the PDF for values of $z$ smaller than $R$ is zero. For convenience, the Hermitian conjugate operator $\widehat{\mathcal{L}}^{\dagger}$ of $\widehat{\mathcal{L}}$ with respect to the un-weighted inner-product is introduced,

$$
\int_{z>R} d \mathbf{r} g(\mathbf{r}) \widehat{\mathcal{L}} f(\mathbf{r})=\int_{z>R} d \mathbf{r} f(\mathbf{r}) \widehat{\mathcal{L}}^{\dagger} g(\mathbf{r}) .
$$

Equation 25 can be rewritten in terms of this Hermitian operator as,

$$
g_{1}(t) \propto \int_{z_{0}>R} d \mathbf{r}_{0} \int_{z>R} d \mathbf{r} \delta\left(\mathbf{r}-\mathbf{r}_{0}\right) \exp \left\{\widehat{\mathcal{L}}^{\dagger} t\right\} P\left(\mathbf{r}_{0}\right) \exp \left\{\imath \mathbf{Q} \cdot\left(\mathbf{r}_{0}-\mathbf{r}\right)\right\} \exp \left\{-\frac{\kappa}{2}\left(z_{0}+z\right)\right\} .
$$


Since $\widehat{\mathcal{L}}^{\dagger}$ is not acting on $\mathbf{r}_{0}$ and $z_{0}$, the terms containing these variables alone can be taken in front of the operator exponential, and the integration with respect to $\mathbf{r}_{0}$ can be performed to yield,

$$
g_{1}(t) \propto \int_{z>R} d \mathbf{r} P(\mathbf{r}) \exp \{\imath \mathbf{Q} \cdot \mathbf{r}\} \exp \left\{-\frac{\kappa z}{2}\right\} \exp \left\{\widehat{\mathcal{L}}^{\dagger} t\right\} \exp \{-\imath \mathbf{Q} \cdot \mathbf{r}\} \exp \left\{-\frac{\kappa z}{2}\right\} .
$$

The short-time behaviour of this correlation function can be obtained by truncation of the Taylor series that defines the operator-exponential after the two leading time-dependent terms, that is, $\exp \left\{\widehat{\mathcal{L}}^{\dagger} t\right\} \approx \hat{I}+\widehat{\mathcal{L}}^{\dagger} t+\widehat{\mathcal{L}}^{\dagger} \widehat{\mathcal{L}}^{\dagger} t^{2} / 2$ (with $\hat{I}$ the identity operator). For short times, eq. 29 can thus be written as,

$$
g_{1}(t)=\left(1+\Gamma t+\frac{\Gamma_{2} t^{2}}{2}\right) \approx \exp \left\{\Gamma t+\frac{\Gamma_{2} t^{2}}{2}\right\}
$$

where,

$$
\Gamma=\frac{\int_{z>R} d \mathbf{r} P(\mathbf{r}) \exp \{\imath \mathbf{Q} \cdot \mathbf{r}\} \exp \left\{-\frac{\kappa z}{2}\right\} \widehat{\mathcal{L}}^{\dagger}\left[\exp \{-\imath \mathbf{Q} \cdot \mathbf{r}\} \exp \left\{-\frac{\kappa z}{2}\right\}\right]}{\int_{z>R} d \mathbf{r} P(\mathbf{r}) \exp \{-\kappa z\}} .
$$

It is used here that $g_{1}$ is unity at time $t=0$.

The explicit form for the Hermitian conjugate operator can be found from its definition in eq. 27 by partial integrations, noting that both $D_{\perp, \|}(z)$ vanish when the colloidal sphere touches the wall (for $z=R$ ) and that $d V / d z$ vanishes at infinity, together with the form 23 for the operator $\widehat{\mathcal{L}}$,

$$
\widehat{\mathcal{L}}^{\dagger}(\cdots)=\frac{\partial}{\partial z}\left(D_{\perp}(z) \frac{\partial}{\partial z}(\cdots)\right)+D_{\|}(z)\left(\frac{\partial^{2}}{\partial x^{2}}+\frac{\partial^{2}}{\partial y^{2}}\right)(\cdots)-D_{\perp}(z) \beta\left[\frac{d V(z)}{d z}\right] \frac{\partial}{\partial z}(\cdots),
$$

where the rectangular brackets on $d V / d z$ are used to indicate that the $z$-differentiation is limited to $V$.

Substitution of this result into eq. 31, leads, after straight forward differentiation and partial integrations, to,

$$
\Gamma=\frac{\int_{z>R} \exp \{-\beta V(z)\} \exp \{-\kappa z\}\left[D_{\|}(z) Q_{\|}^{2}+D_{\perp}(z)\left(Q_{\perp}^{2}+\frac{\kappa^{2}}{4}\right)\right]}{\int_{z>R} \exp \{-\beta V(z)\} \exp \{-\kappa z\}} .
$$

Note that the $x, y$-integrations render both integrals in eq. 31 proportional to the area of illumination of the sample, which thus cancels out.

In case there is no potential in addition to the hard-core potential between the wall and the colloidal sphere, that is, when $V(z) \equiv 0$, eq. 33 reduces to,

$$
\Gamma=\kappa \int_{R}^{\infty} d z \exp \{-\kappa(z-R)\}\left[D_{\|}(z) Q_{\|}^{2}+D_{\perp}(z)\left(Q_{\perp}^{2}+\frac{\kappa^{2}}{4}\right)\right] .
$$


This is the expression for the first cumulant for particles which have no interaction with the wall other than excluded-volume interactions.

The next higher term of expansion 30 is equal to

$$
\Gamma_{2}=\frac{\int_{z>R} d \mathbf{r} P(\mathbf{r}) \exp \{\imath \mathbf{Q} \cdot \mathbf{r}\} \exp \left\{-\frac{\kappa z}{2}\right\} \widehat{\mathcal{L}}^{\dagger} \widehat{\mathcal{L}}^{\dagger}\left[\exp \{-\imath \mathbf{Q} \cdot \mathbf{r}\} \exp \left\{-\frac{\kappa z}{2}\right\}\right]}{\int_{z>R} d \mathbf{r} P(\mathbf{r}) \exp \{-\kappa z\}} .
$$

Using the Hermitian conjugate operator of eq. 32 it is rather straight forward thought tedious to calculate the second cumulant as

$$
\begin{aligned}
& \Gamma_{2} \int_{z>R}^{\infty} d \mathbf{r} \exp \{-\beta V(z)\} \exp \{-\kappa z\}=-\int_{z>R}^{\infty} d \mathbf{r} \exp \{-\beta V(z)\} \exp \{-\kappa z\} \\
&\left\{-\left(D_{\|}(z) Q_{\|}^{2}\right)^{2}-\left(D_{\perp}(z)\left(Q_{\perp}^{2}+\frac{\kappa^{2}}{4}\right)\right)^{2}+2 D_{\|}(z) D_{\perp}(z) Q_{\|}^{2}\left(\frac{\kappa^{2}}{4}-Q_{\perp}^{2}\right)\right. \\
&+\left(\frac{d D_{\perp}(z)}{d z}-D_{\perp}(z) \beta \frac{d V(z)}{d z}\right) \times \kappa\left[D_{\|}(z) Q_{\|}^{2}+D_{\perp}(z)\left(Q_{\perp}^{2}+\frac{\kappa^{2}}{4}\right)\right] \\
&\left.-\left(\frac{d D_{\perp}(z)}{d z}-D_{\perp}(z) \beta \frac{d V(z)}{d z}\right)^{2} \times\left(Q_{\perp}^{2}+\frac{\kappa^{2}}{4}\right)\right\} .
\end{aligned}
$$

If the interaction between the colloidal sphere and the wall is a hard-core interaction, this simplifies to

$$
\begin{aligned}
\Gamma_{2}=-\kappa \int_{R}^{\infty} d z \exp \{-\kappa(z-R)\} \times\{ & -\left(D_{\|}(z) Q_{\|}^{2}\right)^{2}-\left(D_{\perp}(z)\left(Q_{\perp}^{2}+\frac{\kappa^{2}}{4}\right)\right)^{2} \\
& +2 D_{\|}(z) D_{\perp}(z) Q_{\|}^{2}\left(\frac{\kappa^{2}}{4}-Q_{\perp}^{2}\right) \\
& +\frac{d D_{\perp}(z)}{d z} \times \kappa\left[D_{\|}(z) Q_{\|}^{2}+D_{\perp}(z)\left(Q_{\perp}^{2}+\frac{\kappa^{2}}{4}\right)\right] \\
& \left.-\left(\frac{d D_{\perp}(z)}{d z}\right)^{2} \times\left(Q_{\perp}^{2}+\frac{\kappa^{2}}{4}\right)\right\} .
\end{aligned}
$$

This is the expression, which was used in the present paper to estimate the maximum time up to which a first cumulant approximation of the scattered field auto-correlation function is reasonable.

\section{References}

1 K. H. Lan, N. Ostrowsk, and D. Sornette, Phys. Rev. Lett. 57, 17 (1986). 
2 H. A. Lorentz, Abh. Theor. Phys. 1, 23 (1907).

3 H. Faxén, Ark. Mat. Astrom. Fys. 17, 1 (1923).

4 H. Brenner, Chem. Eng. Sci. 16, 242 (1961).

5 A. J. Goldman, R. G. Cox and H. Brenner Chem. Eng. Sci. 22, 637 (1967).

6 V. D. Hopper and A. M. Grant Aust. J. Res. A1, 28 (1948).

7 G. E. Charles and S. G. Mason J. Colloid. Sci. 15, 105 (1960).

8 G. D. MacKay and S. G. Mason J. Colloid. Sci. 16, 632 (1961).

9 Z. Adamcyzk, M. Adamcyzk and T. G. M. Van De Ven J. Colloid Interface Sci. 96, 204 (1983).

10 A. Banerjee and K. D. Kihm, Phys. Rev. E 72, 042101 (2005).

11 K. D. Kihm, A. Banerjee, C. K. Choi and T. Tagaki, Exp. Fluids 37, 8011 (2004).

12 D. C. Prieve, Adv. Colloid Interface. Sci. 82, 93 (1999).

13 D. C. Prieve, F. Luo, and F. Lanni, Faraday Discuss. Chem. Soc. 83, 22 (1987).

14 J. Y. Walz and L. Suresh, J. Chem. Phys. 103, 24 (1995).

15 B. Lin, J. Yu, and S. A. Rice, Phys. Rev. E 62, 3909 (2000).

16 L. Joly, C. Ybert, and L. Bocquet, Phys. Rev. Lett. 96, 046101 (2006).

17 M. I. M. Feitosa and O. M. Mesquita, Phys. Rev. A 44, 6677 (1991).

18 N. Garnier and N. Ostrowsky, J. Phys. II 1, 1221 (191).

19 M. Hosoda, K. Sakai, and K. Tagaki, Phys. Rev. E 58, 6275 (1998).

20 P. Holmqvist, J. K. G. Dhont, and P. R. Lang, Phys. Rev. E 74, 021402 (2006). DOI: 10.1103/PhysRevE.74.021402

21 M. E. O’Neill, Mathematika 11, 67 (1964).

22 E. W. Weisstein, "En-Function." From MathWorld - A Wolfram Web Resource. http://mathworld.wolfram.com/En-Function.html

23 B. Loppinet, G. Petekidis and G. Fytas, Langmuir 14, 4958 (1998).

24 H. Matsuoka, H. Morikawa, S. Tanimoto, A. Kubota, Y. Naito and H. Yamaoka, Colloid Polym. Sci 276, 349 (1998).

25 e. g. J. K. G. Dhont An Introduction to Dynamics of Colloids in D. Möbius and R. Miller (Eds.) Studies in Interface Science, Elsevier, 1996. 


\section{Figure Captions}

Figure 1 Schematic sketch of the instrumental set up and the scattering geometry.

Figure 2 Intensity time-auto correlation functions from the sample with $R=85 \mathrm{~nm}$ recorded at two different penetration depths as indicated in the figure. The scattering vector components are the same for both penetration depths, i. e. $Q_{\|}=0.01316 \mathrm{~nm}^{-1}$ and $Q_{\perp}=0.01145 \mathrm{~nm}^{-1}$. In the inset the initial decay of the field auto correlation functions is displayed. $g_{1}(t)$ is calculated from the experimental data with eq. 18. For comparison the bulk correlation function recorded at the same scattering vector magnitude $Q=\sqrt{Q_{\|}^{2}+Q_{\perp}^{2}}$ is also displayed.

Figure 3 Estimates of the maximum time allowed for a first cumulant approximation of $g_{1}(t)$ as a function of the parallel scattering vector component. The curves have been calculated at constant $Q_{\perp}=0.0114 \mathrm{~nm}^{-1}$ for hard spheres of different radii suspended in water using eqs. 34 and 37. Upper group: $R=85 \mathrm{~nm}$, lower group: $R=27 \mathrm{~nm}$, full lines: $2 / \kappa=800$ $\mathrm{nm}$, dashed lines: $2 / \kappa=500 \mathrm{~nm}$ and dotted lines $2 / \kappa=200 \mathrm{~nm}$.

Figure 4 Theoretical predictions for the ratio between mean diffusivities as measured by 
EWDLS and bulk diffusion coefficient as a function of the normalized penetration depth.

Symbols represent approximations according to eqs. $14\left(\left\langle D_{\|}\right\rangle / D_{0}\right.$ open circles $)$ and 15 $\left(\left\langle D_{\perp}\right\rangle / D_{0}\right.$ open squares). Adjacent full lines represent the corresponding exact solution obtained by numerical integration of eq. 13 with a maximum number of terms, $n_{\max }=50$, used for the calculation of $\lambda_{\perp}$ (eq.1). Inset: Variation of the exact expression for $\left\langle D_{\perp}\right\rangle / D_{0}$ with $n_{\max }$ : dotted line: $n_{\max }=1$, dashed line: $n_{\max }=2$, dashed-dotted line: $n_{\max }=10$, full line: $n_{\max }=50$. For comparison the approximation is displayed as open squares.

Figure 5 Initial relaxation rates as determined by non-linear least squares fitting of $g_{2}(t)$ with eq. 19. Correlation functions were recorded from the sample with $R=85 \mathrm{~nm}$ at a penetration depth of $2 / \kappa=234 \mathrm{~nm}$. The symbol $(\bullet)$ represents a set of data points where $Q_{\|}$was scanned at constant $Q_{\perp}$, while the symbol(o) refers to a scan of $Q_{\perp}$ at constant $Q_{\|}$. The straight lines represent the linear least squares fits from which the diffusivities are extracted.

Figure 6 Mean diffusivities of different particles in dependence of normalized penetration depth. The symbols represent experimental data, full symbols: $R=85 \mathrm{~nm}$, open symbols: $R=27 \mathrm{~nm}$, squares: $\left\langle D_{\|}\right\rangle$and circles: $\left\langle D_{\perp}\right\rangle$. Solid lines are predictions calculated by numerical integration of eq. 13. Broken lines represent theoretical prediction including electrostatic 
interaction between a sphere with $R=85 \mathrm{~nm}$ and the wall with constant Debye screening length $\kappa_{D}^{1}=3 \mathrm{~nm}$ and varying charge parameter: $B_{e s}=10^{4} k_{B} T$ dotted, $B_{e s}=100 k_{B} T$ dashed and $B_{e s}=-7 k_{B} T$ short dashed.

Figure 7 Mean diffusivities of different particles in dependence of normalized penetration depth. The symbols represent experimental data, full symbols: $R=85 \mathrm{~nm}$, open symbols: $R=27 \mathrm{~nm}$, squares: $\left\langle D_{\|}\right\rangle$and circles: $\left\langle D_{\perp}\right\rangle$. Solid lines are predictions calculated by numerical integration of eq. 13. Broken lines represent theoretical prediction including electrostatic interaction between particles and the wall with constant charge parameter $B_{e s}=100 k_{B} T$ and varying Debye screening length : $\kappa_{D}^{1} / R=10$ dashed-dotted, $\kappa_{D}^{1} / R=1$ dotted and $\kappa_{D}^{1} / R=0.05$ dashed. 


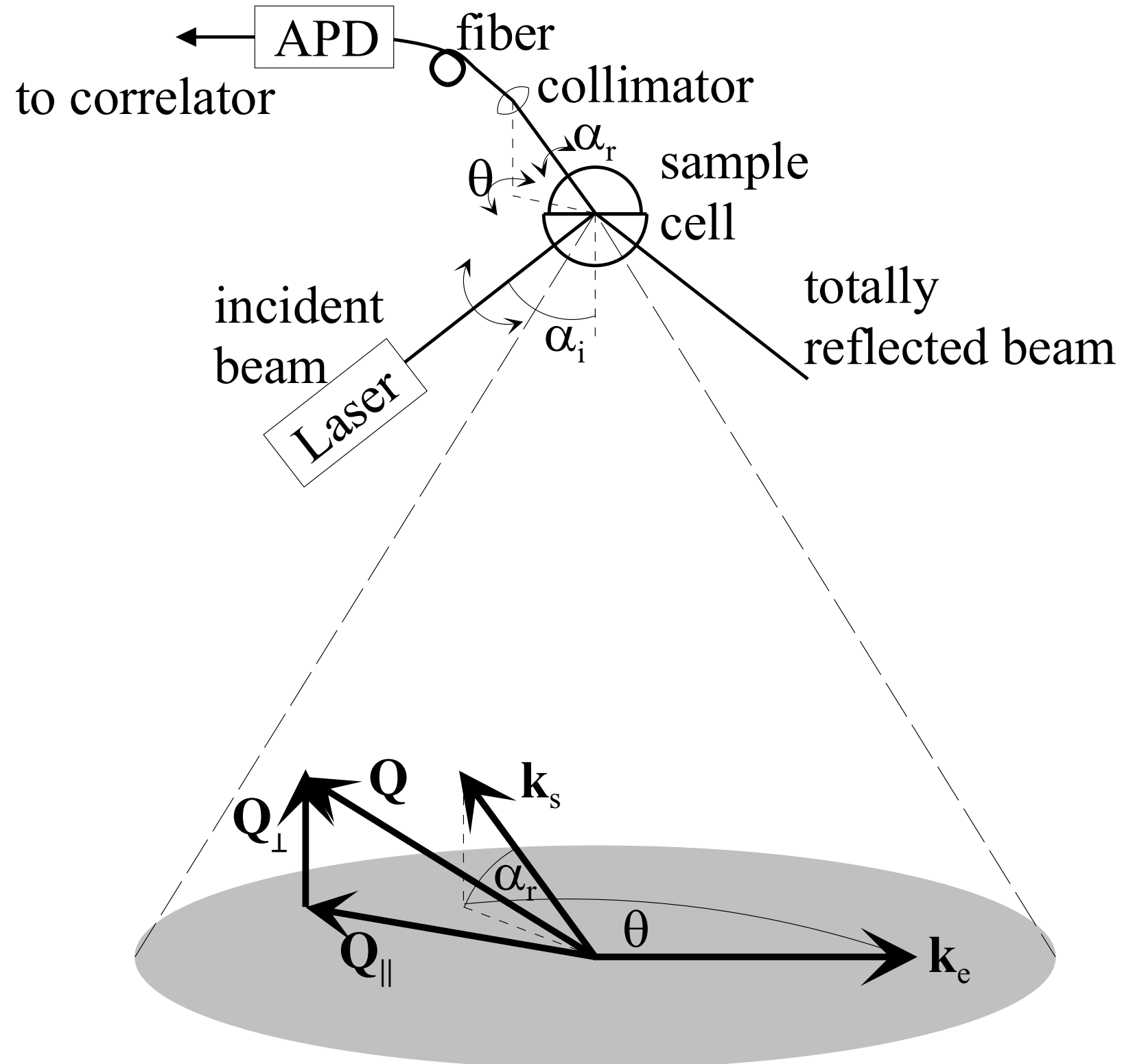

FIG. 1: 


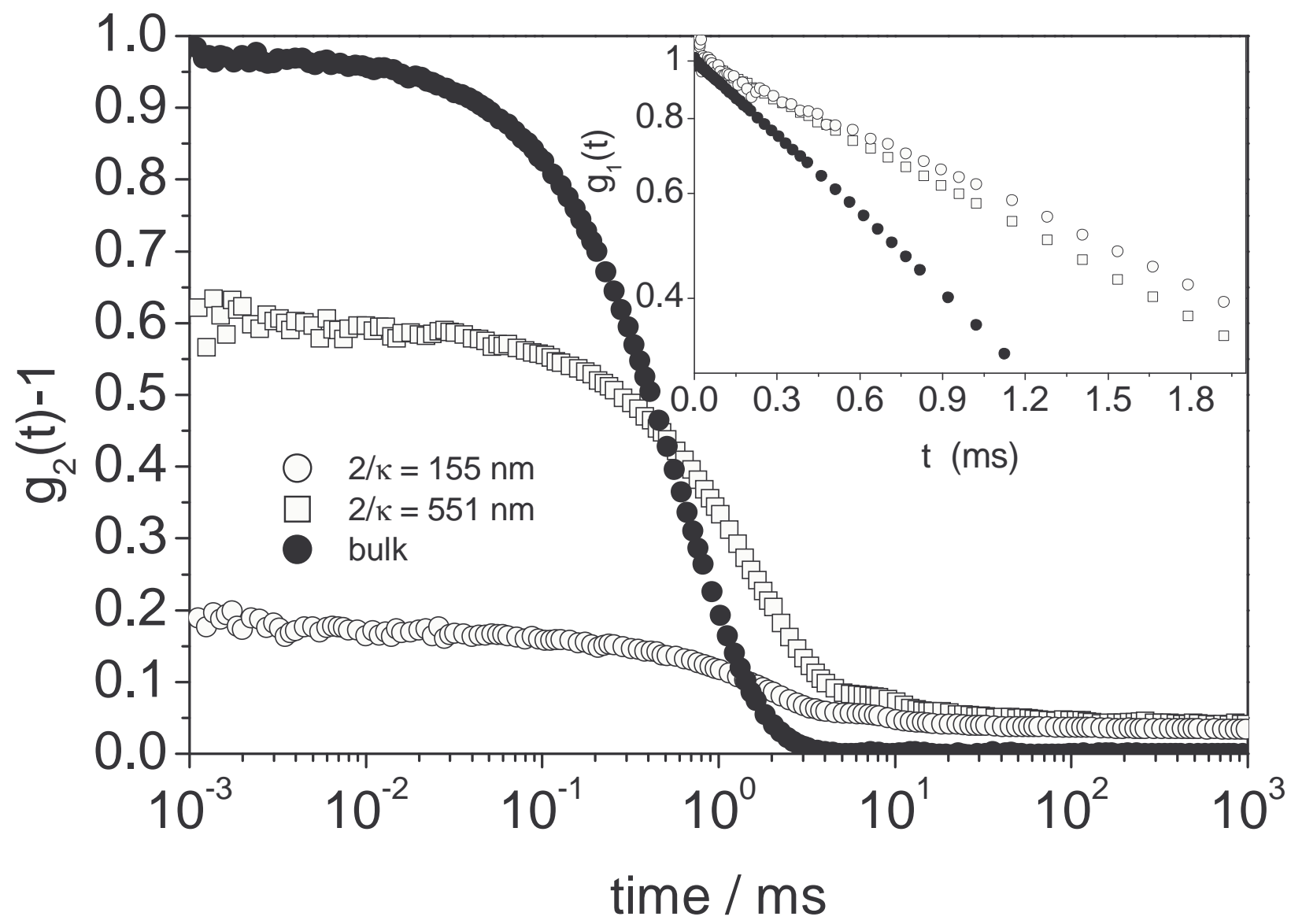

FIG. 2: 


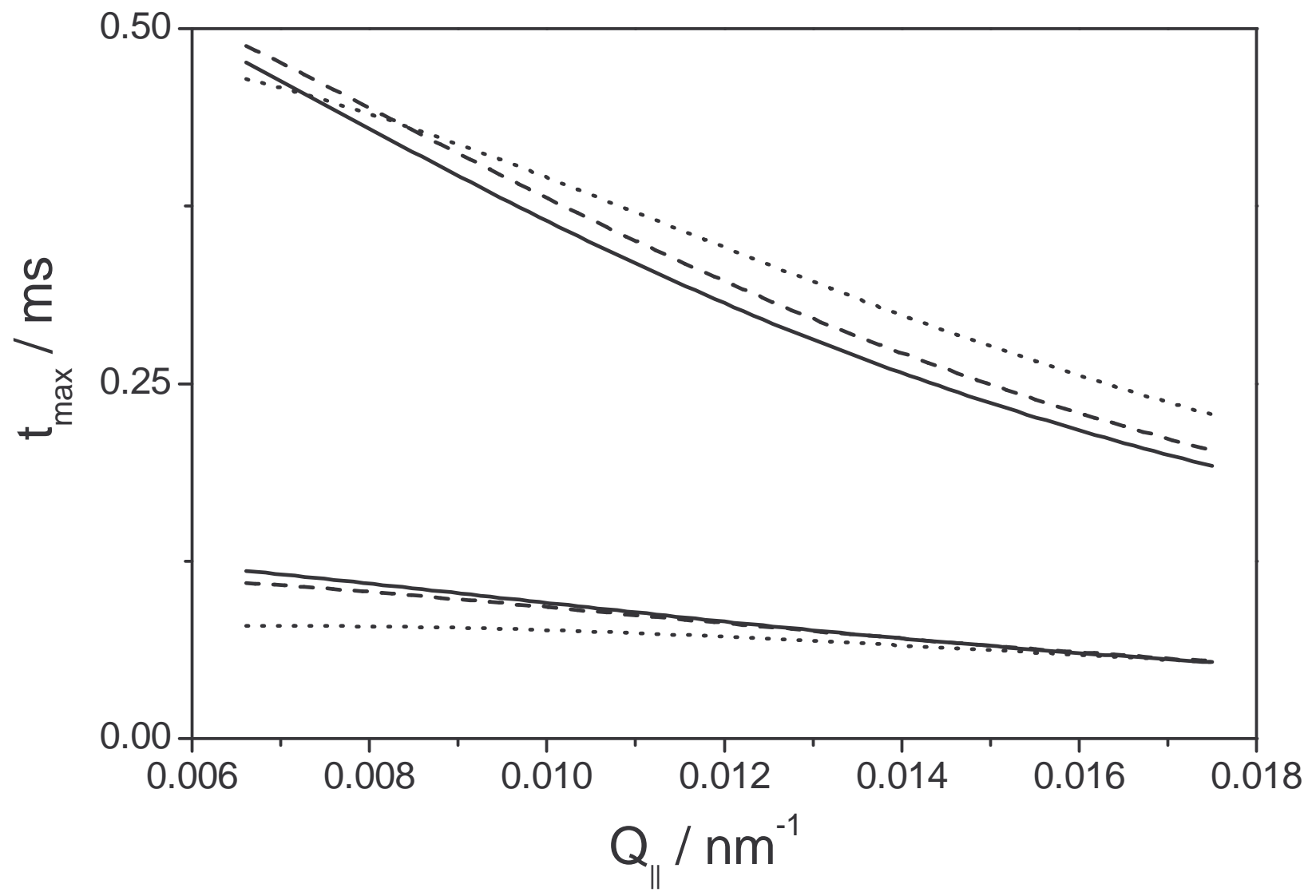

FIG. 3: 


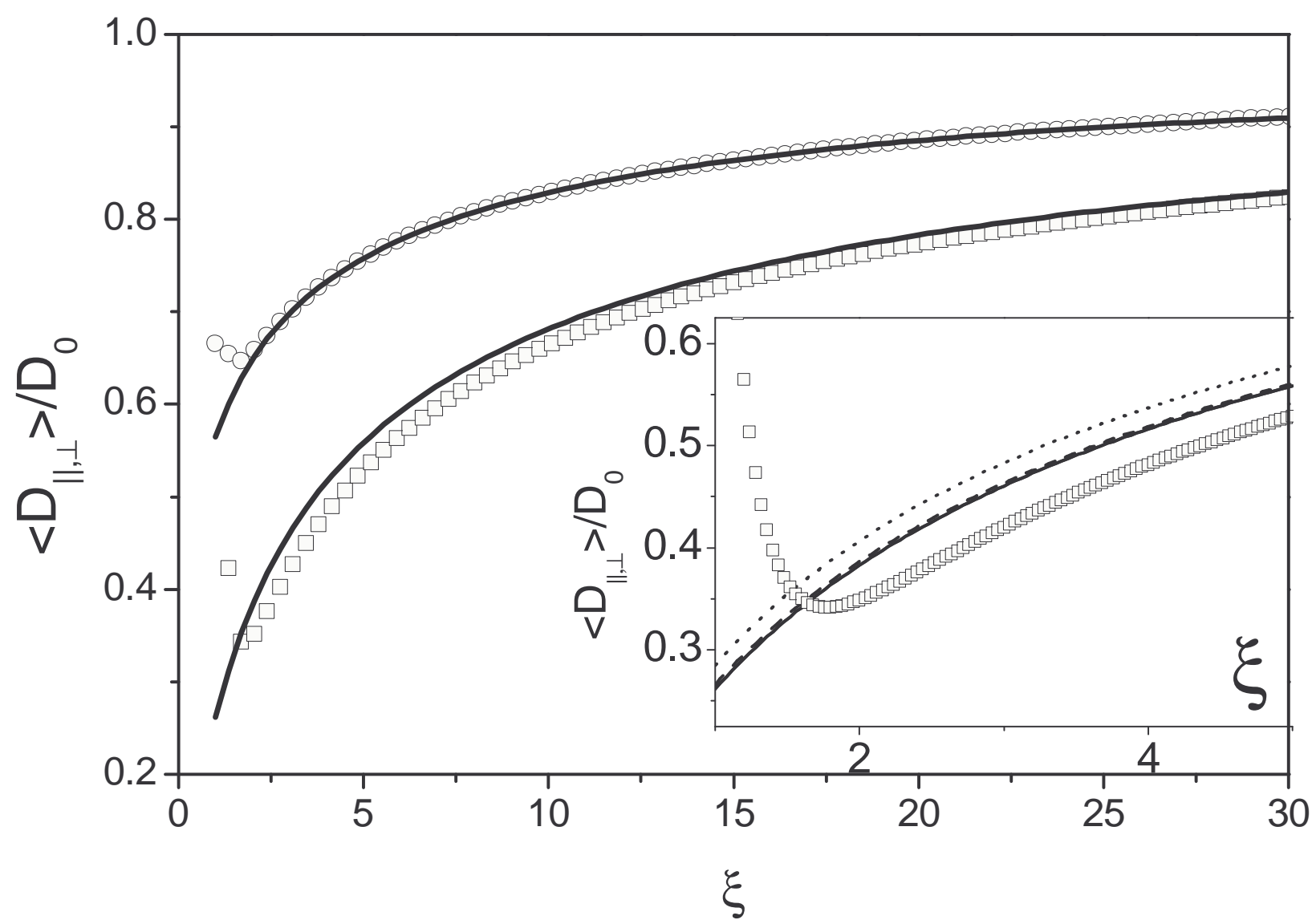

FIG. 4: 


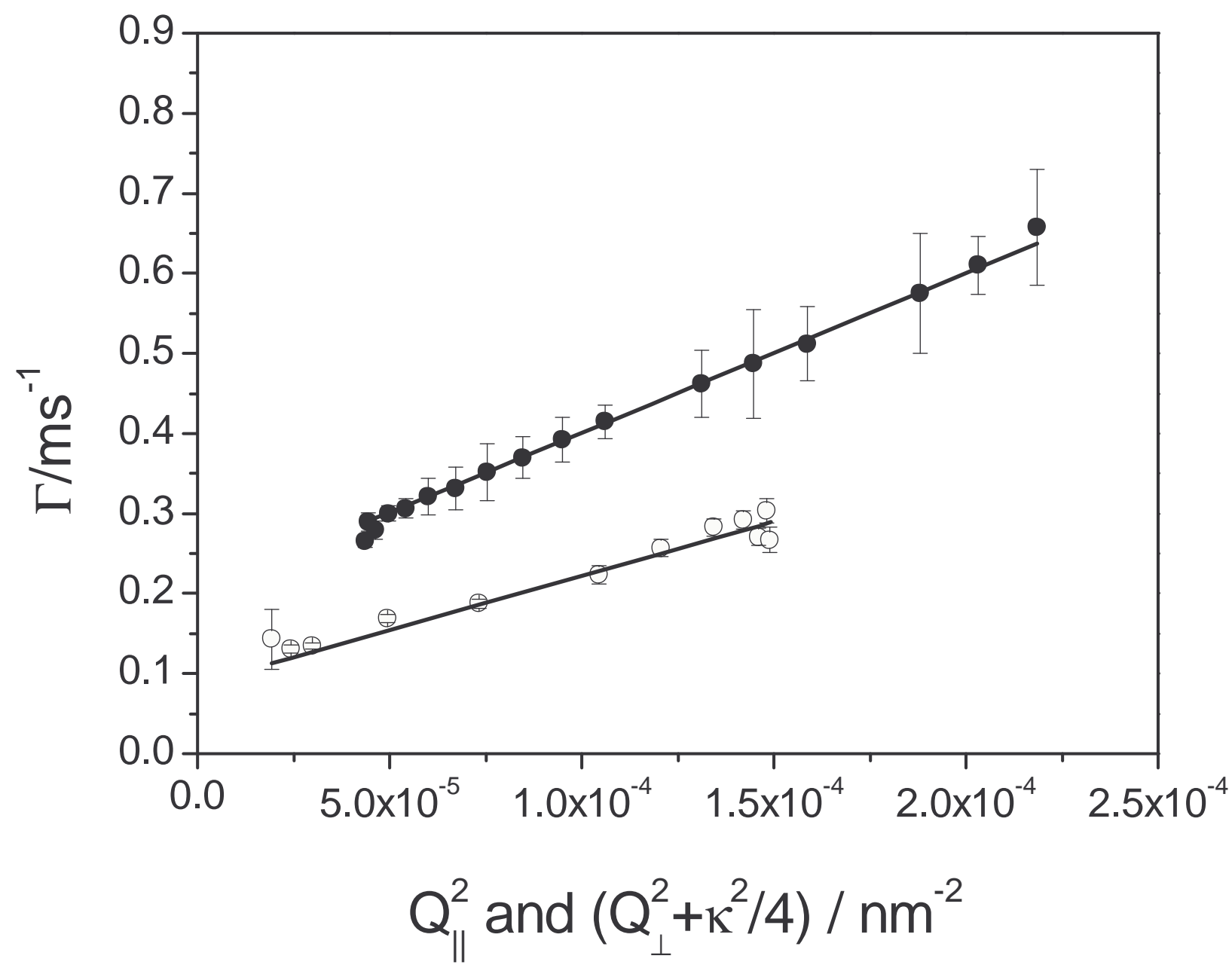

FIG. 5: 


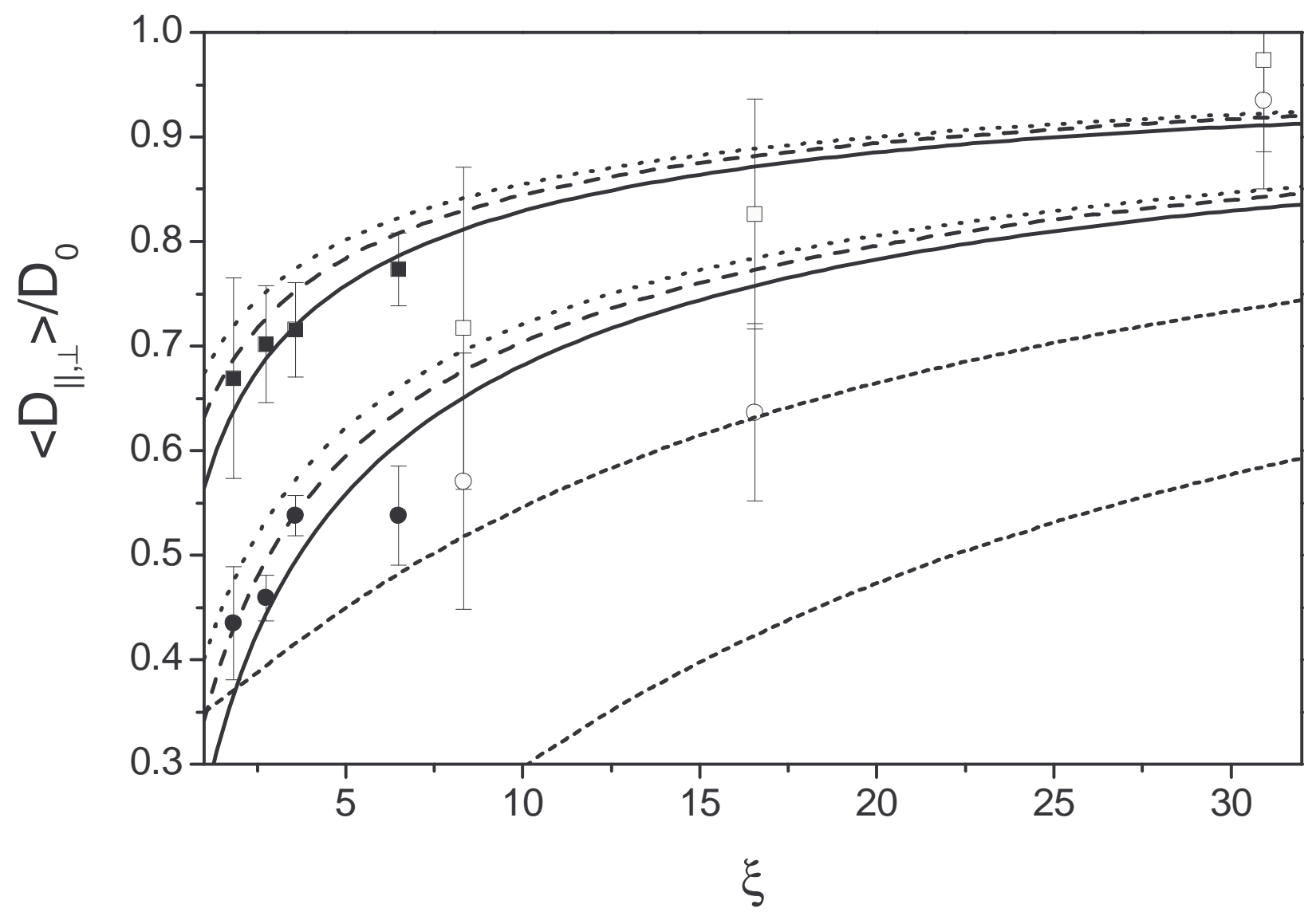

FIG. 6: 


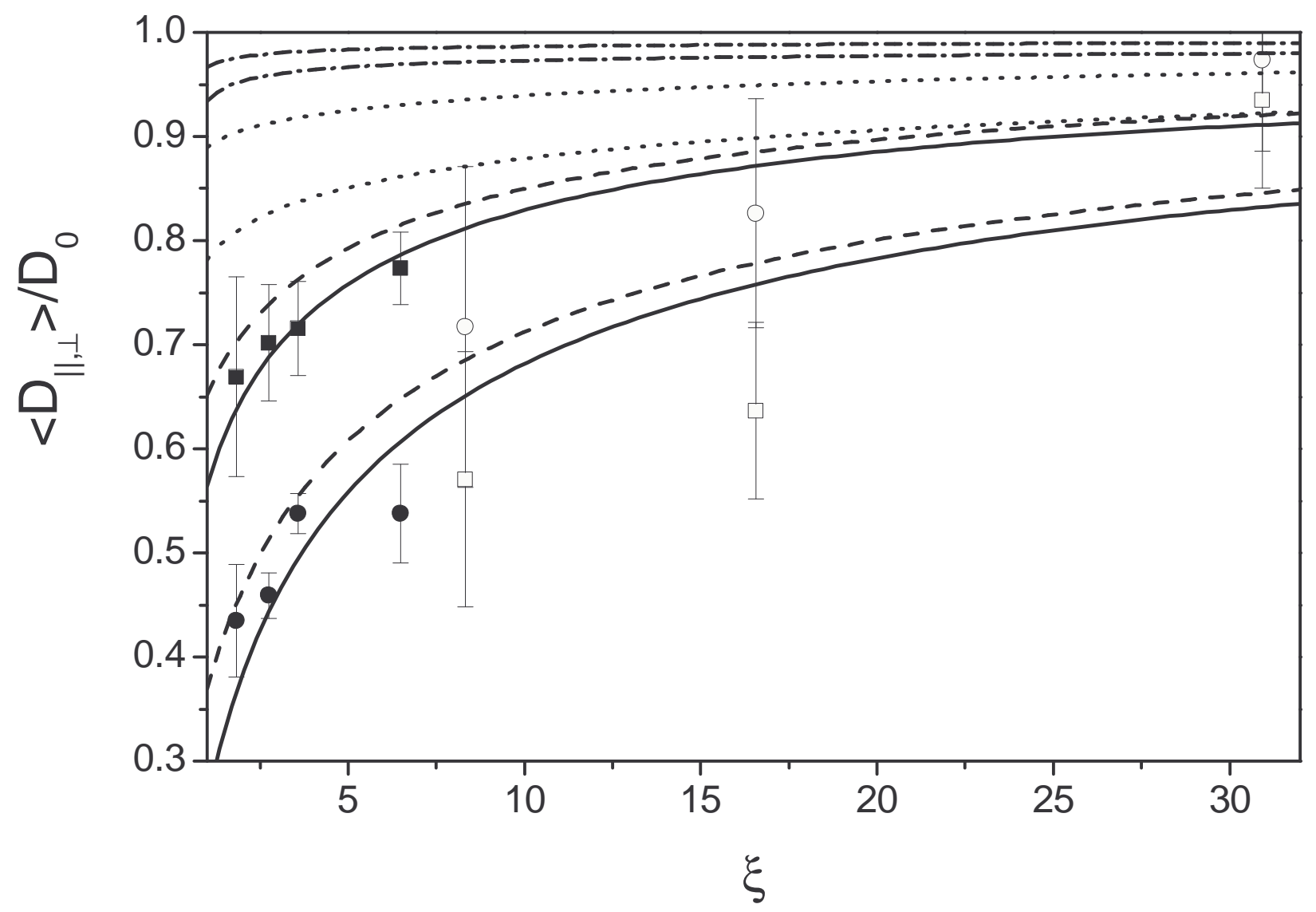

FIG. 7: 